\title{
Coexistence of intrinsic piezoelectricity and ferromagnetism induced by small biaxial strain in septuple-atomic-layer $\mathrm{VSi}_{2} \mathrm{P}_{4}$
}

\author{
San-Dong Guo ${ }^{1}$, Wen-Qi $\mathrm{Mu}^{1}$, Yu-Tong $\mathrm{Zhu}^{1}$ and Xing-Qiu Chen ${ }^{2,3}$ \\ ${ }^{1}$ School of Electronic Engineering, Xi'an University of Posts and Telecommunications, Xi'an 710121, China \\ ${ }^{2}$ Shenyang National Laboratory for Materials Science, Institute of Metal Research, \\ Chinese Academy of Science, 110016 Shenyang, Liaoning, P. R. China and \\ ${ }^{3}$ School of Materials Science and Engineering, University of Science and Technology of China, Shenyang 110016, P. R. China
}

\begin{abstract}
The septuple-atomic-layer $\mathrm{VSi}_{2} \mathrm{P}_{4}$ with the same structure of experimentally synthesized $\mathrm{MoSi}_{2} \mathrm{~N}_{4}$ is predicted to be a spin-gapless semiconductor (SGS). In this work, the biaxial strain is applied to tune electronic properties of $\mathrm{VSi}_{2} \mathrm{P}_{4}$, and it spans a wide range of properties upon the increasing strain from ferromagnetic metal (FMM) to SGS to ferromagnetic semiconductor (FMS) to SGS to ferromagnetic half-metal (FMHM). Due to broken inversion symmetry, the coexistence of ferromagnetism and piezoelectricity can be achieved in $\mathrm{FMS} \mathrm{VSi}_{2} \mathrm{P}_{4}$ with strain range of $0 \%$ to $4 \%$. The calculated piezoelectric strain coefficients $d_{11}$ for $1 \%, 2 \%$ and $3 \%$ strains are $4.61 \mathrm{pm} / \mathrm{V}, 4.94$ $\mathrm{pm} / \mathrm{V}$ and $5.27 \mathrm{pm} / \mathrm{V}$, respectively, which are greater than or close to a typical value of $5 \mathrm{pm} / \mathrm{V}$ for bulk piezoelectric materials. Finally, similar to $\mathrm{VSi}_{2} \mathrm{P}_{4}$, the coexistence of piezoelectricity and ferromagnetism can be realized by strain in the $\mathrm{VSi}_{2} \mathrm{~N}_{4}$ monolayer. Our works show that $\mathrm{VSi}_{2} \mathrm{P}_{4}$ in FMS phase with intrinsic piezoelectric properties can have potential applications in spin electronic devices.
\end{abstract}

PACS numbers: 71.20.-b, 77.65.-j, 72.15.Jf, 78.67.-n

Email:sandongyuwang@163.com

Keywords: Ferromagnetism, Piezoelectronics, 2D materials

\section{INTRODUCTION}

Due to the advantages of high speed, high integration density and high power transformers, the twodimensional (2D) magnetic materials have great potential applications for nanoscale spintronic devices ${ }^{1-3}$. A few types of 2D magnetic materials have been studied both in theory and in experiment ${ }^{4-9}$. For example, the $\mathrm{Cr}_{2} \mathrm{Ge}_{2} \mathrm{Te}_{6}$ is an intrinsic ferromagnetic 2D material ${ }^{5}$, and the monolayer $\mathrm{VS}_{2}$ and $\mathrm{VSe}_{2}$ have also been experimentally demonstrated to be magnetic ${ }^{6}$. The $\mathrm{Mn}_{2} \mathrm{C}_{6} \mathrm{Se}_{12}$ and $\mathrm{Mn}_{2} \mathrm{C}_{6} \mathrm{~S}_{6} \mathrm{Se}_{6}$ monolayers are predicted to be Dirac SGSs with $100 \%$ spin polarization, high Fermi velocities and high Curie temperatures ${ }^{7}$. Another particularly interesting property of $2 \mathrm{D}$ materials is piezoelectricity, which is used for energy conversion between electrical and mechanical energy. It has been theoretically reported that many kinds of $2 \mathrm{D}$ materials have significant piezoelectric coefficients ${ }^{10-16}$. Experimentally discovered piezoelectricity (such as $\mathrm{MoS}_{2}{ }^{17,18}, \mathrm{MoSSe}^{19}$ and $\mathrm{In}_{2} \mathrm{Se}_{3}{ }^{20}$ ) has promoted the huge studies on piezoelectric properties of $2 \mathrm{D}$ materials.

It is interesting and useful to combine the piezoelectricity and magnetism into the same kind of 2D material for applications in nanoscale spin electronic devices. The progress has been achieved in $2 \mathrm{D}$ vanadium dichalcogenides, and the $\mathrm{VS}_{2}, \mathrm{VSe}_{2}$ and Janus-VSSe are not only magnetic semiconductors but also exhibit appreciable piezoelectricity ${ }^{21}$. Recently, the layered $2 \mathrm{D} \mathrm{MoSi}_{2} \mathrm{~N}_{4}$ and $\mathrm{WSi}_{2} \mathrm{~N}_{4}$ have been synthesized by chemical vapor deposition $(\mathrm{CVD})^{22}$. And then a new kind of $2 \mathrm{D}$ family $\mathrm{MA}_{2} \mathrm{Z}_{4}$ is proposed with $\alpha_{i}$ and $\beta_{i}$ ( $i=1$ to 6 ) phases by intercalating $\mathrm{MoS}_{2}$-type $\mathrm{MZ}_{2}$ monolayer into InSe-type $\mathrm{A}_{2} \mathrm{Z}_{2}$ monolayer ${ }^{22,23}$. Due to lacking inversion symmetry of $\mathrm{MA}_{2} \mathrm{Z}_{4}$ with $\alpha_{i}$ phase, the piezoelectricity can exist, such as experimentally synthesized $\mathrm{MoSi}_{2} \mathrm{~N}_{4}$ and $\mathrm{WSi}_{2} \mathrm{~N}_{4}$ with $\alpha_{1}$ phase $^{24}$. The $\alpha_{1}-\mathrm{VSi}_{2} \mathrm{P}_{4}$ is predicted to be a $\mathrm{SGS}^{23}$, which may be easily tuned into FMS by strain. This will provide a platform to realize the coexistence of piezoelectricity and magnetism.

In this work, the biaxial strain effects on electronic properties of monolayer $\alpha_{1}-\mathrm{VSi}_{2} \mathrm{P}_{4}$ are studied by the first principle calculations. In considered strain range, the ferromagnetic (FM) ground state of $\mathrm{VSi}_{2} \mathrm{P}_{4}$ is confirmed, and it can change from FMM to SGS to FMS to SGS to FMHM with increasing strain. In FMS phase of $\mathrm{VSi}_{2} \mathrm{P}_{4}$, the piezoelectricities are investigated, and the calculated $d_{11}$ for $1 \%, 2 \%$ and $3 \%$ strains are $4.61 \mathrm{pm} / \mathrm{V}$, $4.94 \mathrm{pm} / \mathrm{V}$ and $5.27 \mathrm{pm} / \mathrm{V}$, respectively. Similar straininduced phase transition can also be achieved in monolayer $\mathrm{VSi}_{2} \mathrm{~N}_{4}$, and only the critical point of phase transition is different from one of $\mathrm{VSi}_{2} \mathrm{P}_{4}$. Our calculations show that the monolayer $\alpha_{1}-\mathrm{VSi}_{2} \mathrm{P}_{4}$ may be promising candidate for spintronic and piezoelectric applications by strain engineering.

The rest of the paper is organized as follows. In the next section, we shall give our computational details and methods. In the third section, we shall present main results of septuple-atomic-layer $\mathrm{VSi}_{2} \mathrm{P}_{4}$. Finally, we shall give our discussion and conclusions in the fourth section.

\section{COMPUTATIONAL DETAIL}

Calculations are based on spin-polarized density functional theory $(\mathrm{DFT})^{25}$ using the popular generalized gradient approximation of Perdew, Burke and Ernzerhof $(\text { GGA-PBE })^{26}$ as the exchange-correlation functional. 


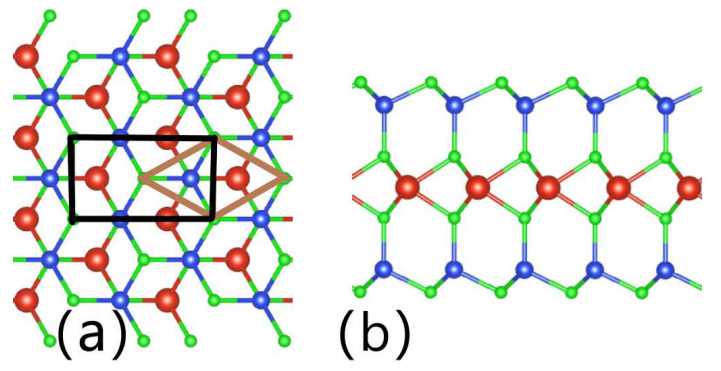

FIG. 1. (Color online)The (a) top view and (b) side view of crystal structure of monolayer $\mathrm{VSi}_{2} \mathrm{P}_{4}$. The large red balls represent $\mathrm{V}$ atoms, and the middle blue balls for $\mathrm{Si}$ atoms, and the small green balls for $\mathrm{P}$ atoms. The primitive cell and rectangle supercell are marked by brown and black lines, respectively.
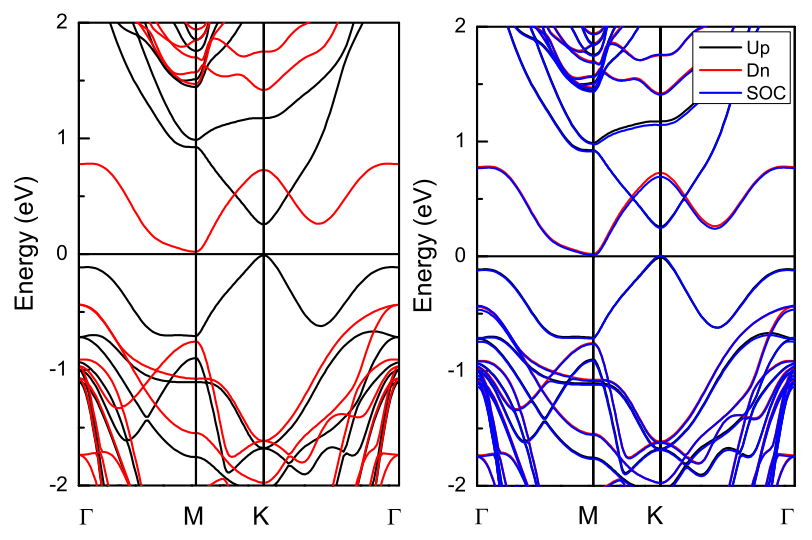

FIG. 2. (Color online)The energy band structures of unstrained $\mathrm{VSi}_{2} \mathrm{P}_{4}$, include using GGA (Left) or GGA+SOC and GGA (Right) with FM state.

Projector augmented wave (PAW) potentials are used in all calculations, as implemented in the plane-wave code VASP $^{27-29}$. A vacuum spacing of more than $32 \AA$ is used in the direction normal to the $2 \mathrm{D}$ monolayer in order to avoid interactions between two neighboring images. The kinetic energy cutoff is set to $500 \mathrm{eV}$, and the total energy convergence criterion is set to $10^{-8} \mathrm{eV}$. The ionic relaxation is performed until the force on each atom is less than $0.0001 \mathrm{eV} . \AA^{-1}$. Piezoelectricity is studied by using density functional perturbation theory $(\mathrm{DFPT})^{30}$. The reciprocal space is represented by the Monkhorst-Pack special k-point scheme with $15 \times 15 \times 1$ meshes for the calculations of electronic structure and elastic coefficients $C_{i j}$, and $9 \times 16 \times 1$ grid meshes for the energy of different magnetic configurations and piezoelectric stress coefficients $e_{i j}$. The 2D elastic coefficients $C_{i j}^{2 D}$ and piezoelectric stress coefficients $e_{i j}^{2 D}$ have been renormalized by $C_{i j}^{2 D}=L z C_{i j}^{3 D}$ and $e_{i j}^{2 D}=L z e_{i j}^{3 D}$, where the $L z$ is the length of unit cell along $\mathrm{z}$ direction.

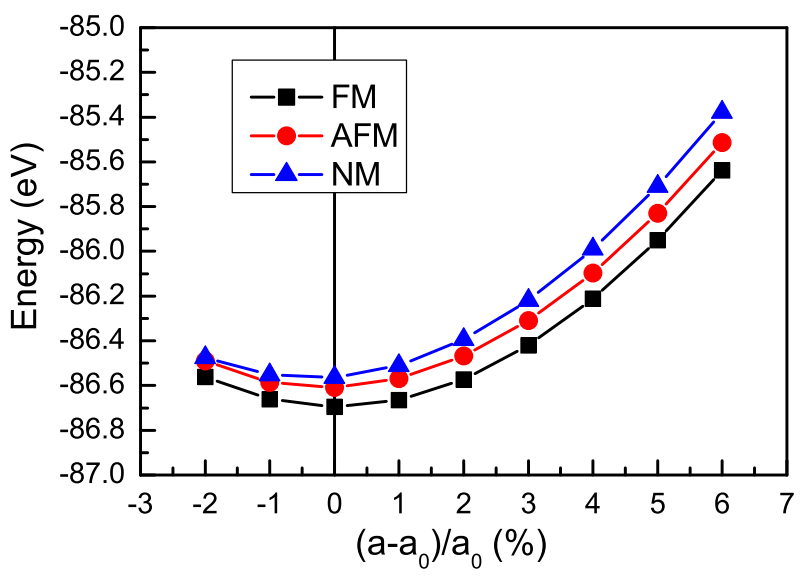

FIG. 3. (Color online) Calculated energy of FM state, AFM state and NM state as a function of strain with rectangle supercell.

\section{MAIN CALCULATED RESULTS}

The $\alpha_{1}-\mathrm{VSi}_{2} \mathrm{P}_{4}$ is predicted to be a parabolic FM SGS with the total magnetic moment $1.0 \mu_{B}{ }^{23}$. Firstly, we relax the geometric structures of $\mathrm{VSi}_{2} \mathrm{P}_{4}$ with spinpolarized calculations, which is plotted in Figure 1. The seven atomic layers of $\mathrm{N}-\mathrm{Si}-\mathrm{N}-\mathrm{V}-\mathrm{N}-\mathrm{Si}-\mathrm{N}$ are observed with a $\mathrm{VN}_{2}$ layer sandwiched between two $\mathrm{Si}-\mathrm{N}$ bilayers. The optimized lattice constants is $3.486 \AA$, which is in good agreement with the previous theoretical result ${ }^{23}$. We calculate electronic band structures of $\mathrm{VSi}_{2} \mathrm{P}_{4}$ using GGA and GGA plus spin orbital coupling (SOC), which are plotted in Figure 2. It is clearly seen that the Fermi energy level happens to touch the minority-spin conduction band minimum $(\mathrm{CBM})$ at $\mathrm{M}$ point and the majority-spin valence band maximum (VBM) at K point at the same time, and a SGS is achieved. Calculated results show that the SOC has little effects on energy bands of $\mathrm{VSi}_{2} \mathrm{P}_{4}$, so we use GGA to investigate the role of strain on the electronic structures of $\mathrm{VSi}_{2} \mathrm{P}_{4}$.

Strain is a very effective way to tune the electronic structures, topological properties, transport and piezoelectric properties of $2 \mathrm{D}$ materials ${ }^{31-34}$. The biaxial strain can be simulated by $\left(a-a_{0}\right) / a_{0}$ with $a$ and $a_{0}$ being the strained and unstrained lattice constants. To determine the ground state of strained $\mathrm{VSi}_{2} \mathrm{P}_{4}$, a rectangle supercell (in Figure 1) is used to construct two different initial magnetic configurations, including antiferromagnetic (AFM) and FM states. The energy of FM state, AFM state and non-magnetic (NM) state as a function of strain are shown in Figure 3. It is clearly seen that $\mathrm{VSi}_{2} \mathrm{P}_{4}$ prefers $\mathrm{FM}$ ground state in considered strain range, and the energy difference between AFM and FM states increases from $73 \mathrm{meV}$ to $124 \mathrm{meV}$, when the strain changes from $-2 \%$ to $6 \%$. This means that the applied biaxial strain can effectively enhance the magnetic coupling of $\mathrm{VSi}_{2} \mathrm{P}_{4}$, increasing the Curie temperature.

The energy band structures of $\mathrm{FM} \mathrm{VSi}_{2} \mathrm{P}_{4}$ using GGA 

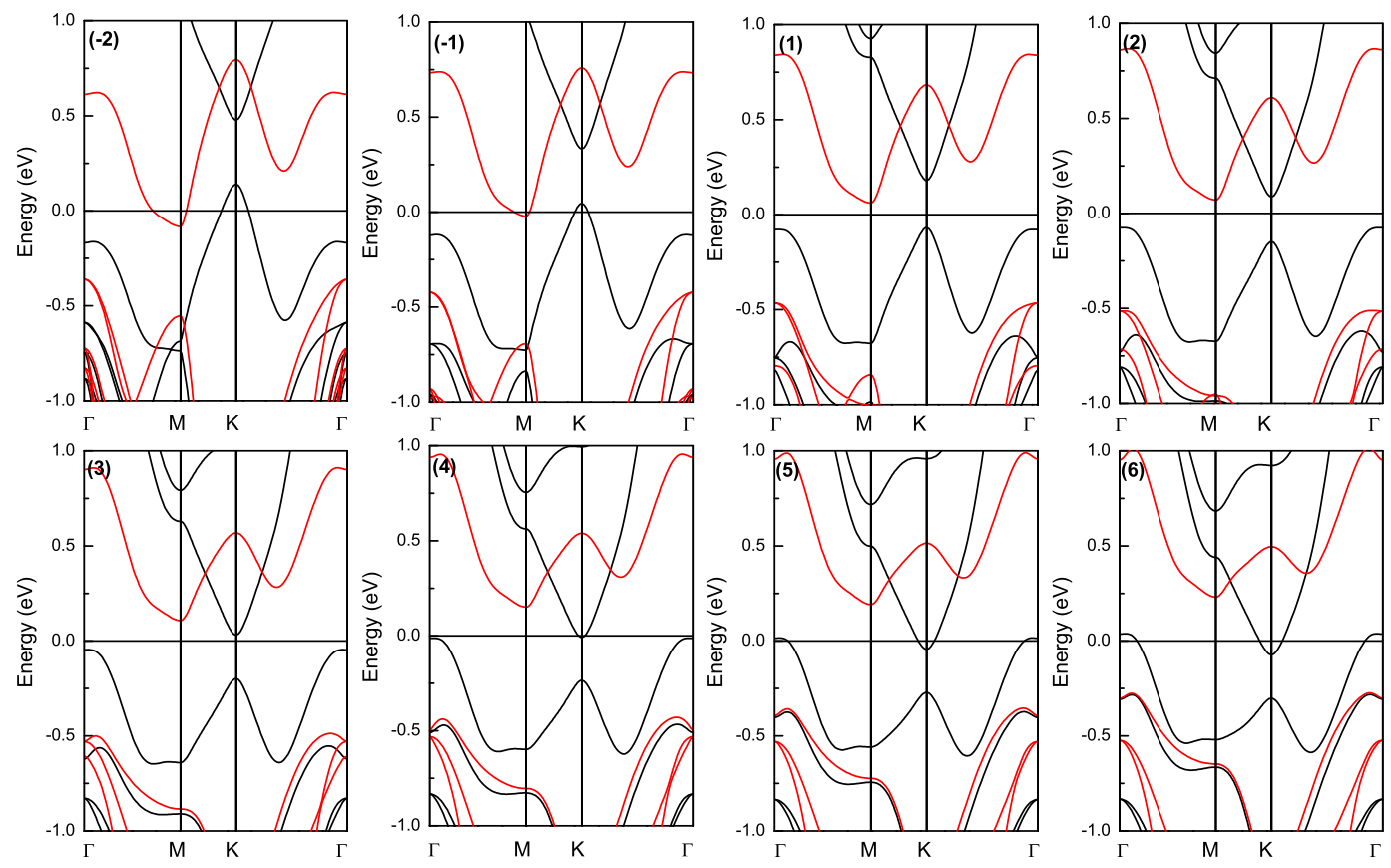

FIG. 4. (Color online) The energy band structures of $\mathrm{FM} \mathrm{VSi}_{2} \mathrm{P}_{4}$ using GGA with strain from $-2 \%$ to $6 \%$ except $0 \%$, which has been shown in Figure 2. The black and red lines represent the spin-up and spin-down bands.

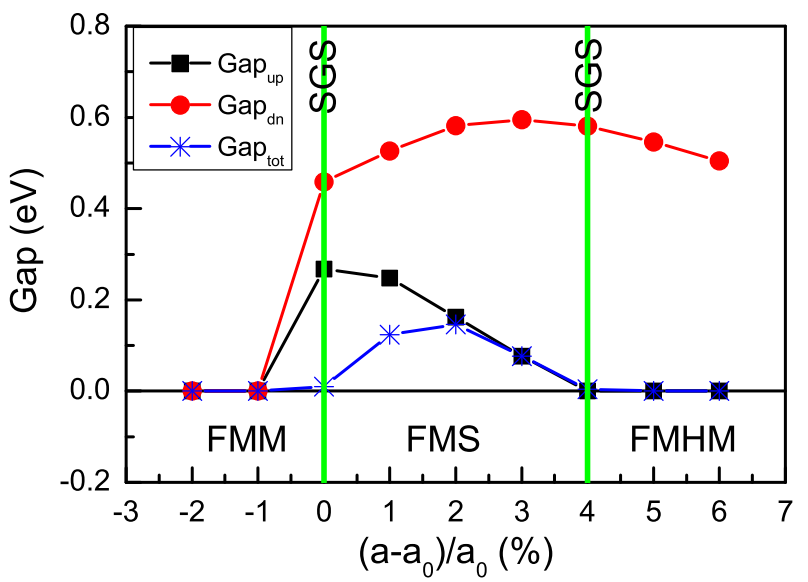

FIG. 5. (Color online)The majority-spin gap (Gapup), the minority-spin gap (Gapdn $)$ and the total gap (Gaptot) of FM $\mathrm{VSi}_{2} \mathrm{P}_{4}$ as a function of strain using GGA. The electronic properties change from FMM to SGS to FMS to SGS to FMHM with the increasing strain.

with strain from $-2 \%$ to $6 \%$ except $0 \%$ are shown in Figure 4 , and the majority-spin, minority-spin and total gaps are plotted in Figure 5. From $-2 \%$ to $6 \%$, the first conduction band of majority-spin moves toward the lower energy, and touches exactly the Fermi level at $4 \%$ strain, and then crosses the the Fermi level at $>4 \%$ strain. The similar trend can be observed for the first conduction band of minority-spin, when the strain changes from $6 \%$ to $-2 \%$, and happen to touch the Fermi level at $0 \%$ strain. For the first valence band of majority-spin, the energy eigenstates around the $\mathrm{K}$ point vary toward lower energy with strain from $-2 \%$ to $6 \%$, and touch the Fermi level at $0 \%$ strain, while the energy eigenstates around the $\Gamma$ point move toward higher energy, and contact the Fermi level at $4 \%$ strain. These lead to diverse properties of $\mathrm{VSi}_{2} \mathrm{P}_{4}$ upon strain from FMM to SGS to FMS to SGS to FMHM. The compressive strain makes both majorityspin and minority-spin gaps be zero, and a FMM is achieved. When the tensile strain is less than $4 \%$, both majority-spin and minority-spin gaps are nonzero, and then the total gap is positive value. In this strain range, a FMS can be induced by tensile strain. With tensile strain being larger than $4 \%$, only minority-spin gap is nonzero, and the majority-spin gap become zero, which gives rise to a FMHM. At the critical states of $0 \%$ and $4 \%$, the SGS can be observed, but they are different. At $0 \%$ strain, there is a gap for both the majority and minority channels, while there is no gap between the majority channel in the valence band and the minority channel in the conduction band. At $4 \%$ strain, the majority channel is gapless, while the minority channel is separated by a gap. Similar stress-tuned SGS can be observed in the ferromagnetic semiconductor $\mathrm{HgCr}_{2} \mathrm{Se}_{4}{ }^{35}$.

The $\mathrm{VSi}_{2} \mathrm{P}_{4}$ has the $\overline{6} m 2$ point group, and then lacks the inversion symmetry, which makes $\mathrm{VSi}_{2} \mathrm{P}_{4}$ to be piezoelectric. A piezoelectric material should be a semiconductor to prohibit current leakage. It is interesting to investigate the piezoelectric properties of $\mathrm{VSi}_{2} \mathrm{P}_{4}$ with the strain range of $0 \%$ to $4 \%$, because the coexistence of piezoelectricity and magnetism can be achieved in the same kind of material. The piezoelectric stress tensors $e_{i j k}$ and strain tensor $d_{i j k}$ can be used to characterize the 

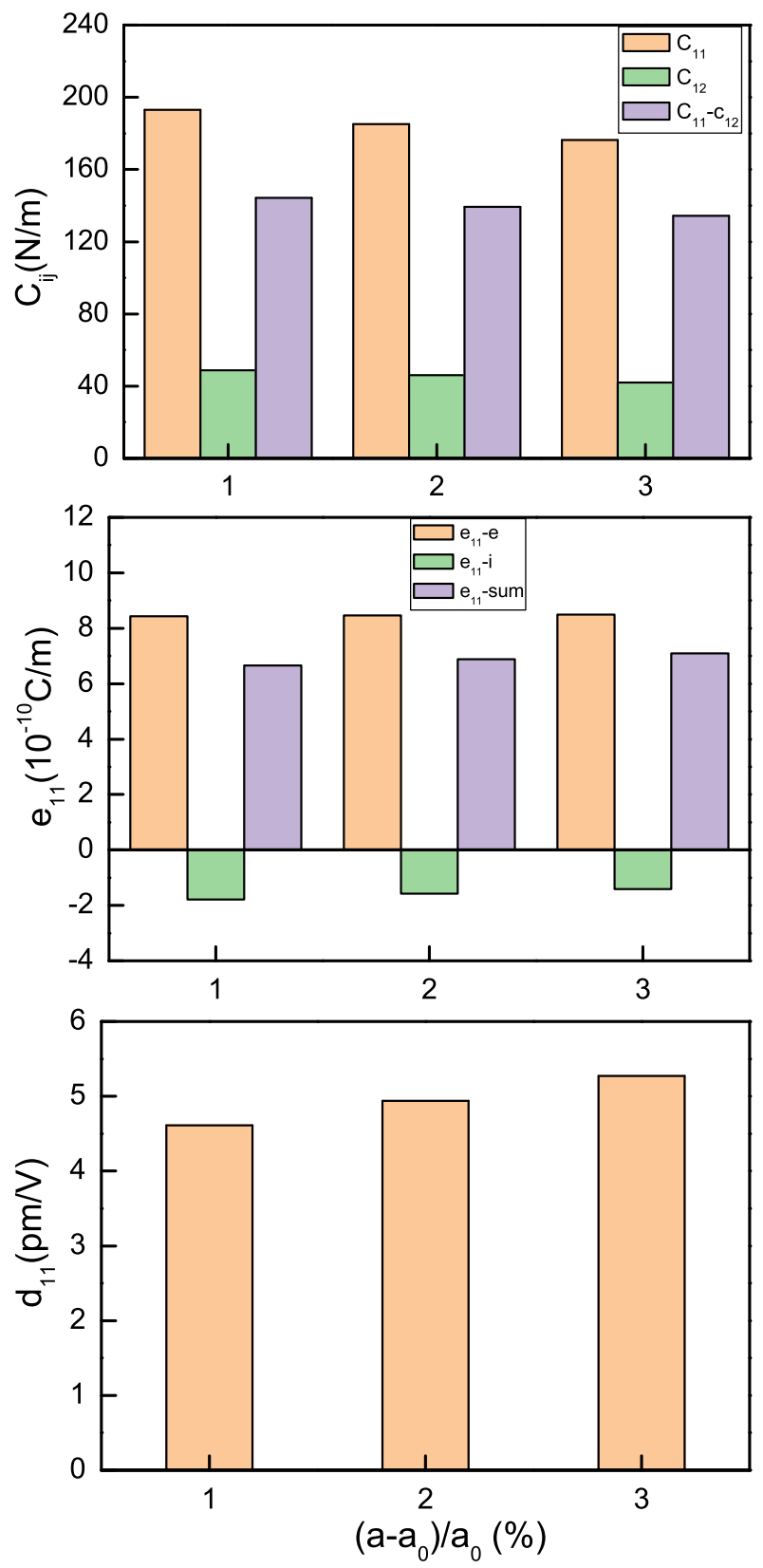

FIG. 6. (Color online)For monolayer $\mathrm{VSi}_{2} \mathrm{P}_{4}$, (Top) the elastic constants $C_{i j}$, (Middle) piezoelectric stress coefficients $e_{11}$ along with the ionic contribution and electronic contribution, and (Bottom) piezoelectric strain coefficients $d_{11}$ with the strain being $1 \%, 2 \%$ and $3 \%$.

linear piezoelectric effect of monolayer $\mathrm{VSi}_{2} \mathrm{P}_{4}$, which include ionic and electronic contributions. Using the Voigt notation, the $e_{i j k}$ and $d_{i j k}$ can be represented as $e_{i j}$ and $d_{i j}$, respectively. The $e_{i k}$ is connected with $d_{i j}$ by:

$$
e_{i k}=d_{i j} C_{j k}
$$

For 2D semiconductors, in general, in-plane stresses and strains are only allowed, while the out-of-plane is strain/stress free ${ }^{10,13,15}$. For $\overline{6} m 2$ point group, the $C_{i j}$,

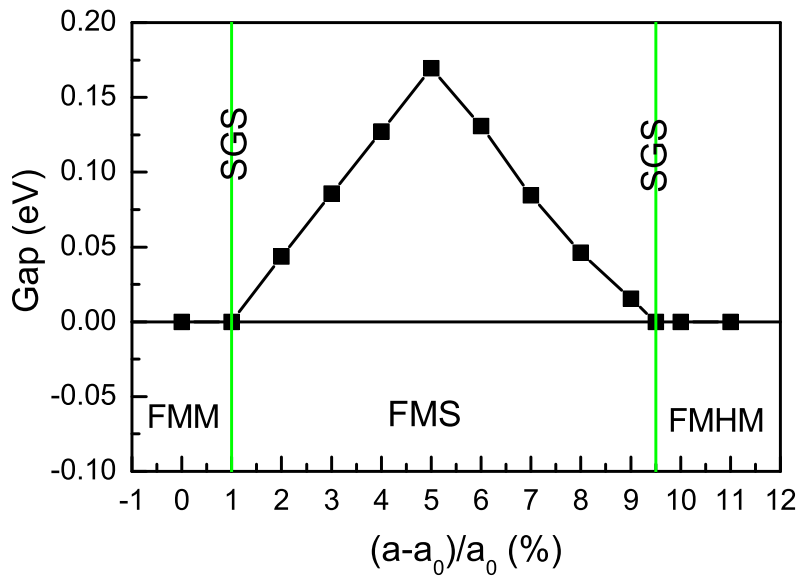

FIG. 7. (Color online)The total gap (Gap) of $\mathrm{FM} \mathrm{VSi}_{2} \mathrm{~N}_{4}$ as a function of strain using GGA. The electronic properties change from FMM to SGS to FMS to SGS to FMHM with the increasing strain.

$e_{i j}$ and $d_{i j}$ of monolayer $\mathrm{VSi}_{2} \mathrm{P}_{4}$ become:

$$
\begin{aligned}
& \left(\begin{array}{ccc}
e_{11} & -e_{11} & 0 \\
0 & 0 & -e_{11} \\
0 & 0 & 0
\end{array}\right) \\
& \left(\begin{array}{ccc}
d_{11} & -d_{11} & 0 \\
0 & 0 & -2 d_{11} \\
0 & 0 & 0
\end{array}\right) \\
& \left(\begin{array}{ccc}
C_{11} & C_{12} & 0 \\
C_{12} & C_{11} & 0 \\
0 & 0 & \frac{C_{11}-C_{12}}{2}
\end{array}\right)
\end{aligned}
$$

Solving the about equations, the only independent inplane $d_{11}$ is:

$$
d_{11}=\frac{e_{11}}{C_{11}-C_{12}}
$$

A rectangle supercell is used for the calculation of $e_{i j}$, as shown in Figure 1. The $C_{i j}$ are calculated by using strain-stress relationship. The elastic constants $C_{i j}$, piezoelectric stress coefficients $e_{11}$ along with the ionic contribution and electronic contribution, and piezoelectric strain coefficients $d_{11}$ of monolayer $\mathrm{VSi}_{2} \mathrm{P}_{4}$ with the strain being $1 \%, 2 \%$ and $3 \%$ are shown in Figure 6 . All calculated elastic coefficients of $\mathrm{VSi}_{2} \mathrm{P}_{4}$ with the strain being $1 \%, 2 \%$ and $3 \%$ satisfy the Born stability criteria ${ }^{36}$. It is found that the ionic and electronic parts have opposite contributions to $e_{11}$. The increasing strain can enhance the $e_{11}$, and these values for $1 \%, 2 \%$ and $3 \%$ strains are $6.65 \times 10^{-10} \mathrm{C} / \mathrm{m}, 6.88 \times 10^{-10} \mathrm{C} / \mathrm{m}$ and $7.09 \times 10^{-10}$ $\mathrm{C} / \mathrm{m}$, respectively. The trend is mainly due to decreased ionic contribution (absolute value). The corresponding values of $d_{11}$ for $1 \%, 2 \%$ and $3 \%$ strains are $4.61 \mathrm{pm} / \mathrm{V}$, 
$4.94 \mathrm{pm} / \mathrm{V}$ and $5.27 \mathrm{pm} / \mathrm{V}$, respectively. The strainimproved $d_{11}$ is due to the enhanced $e_{11}$ and reduced $C_{11}-C_{12}$ according to Equation 5. These results are comparable with or larger than ones of TMD monolayers such as $\mathrm{MoS}_{2}$ obtained from DFT calculations ${ }^{13,15}$. The values of $d_{11}$ of $\mathrm{FMS} \mathrm{VSi}_{2} \mathrm{P}_{4}$ are greater than or close to a typical value for bulk piezoelectric materials, about $5 \mathrm{pm} / \mathrm{V}^{13}$. This indicates that $\mathrm{FMS} \mathrm{VSi}_{2} \mathrm{P}_{4}$ caused by small strain show potential in piezoelectric devices.

The $\mathrm{VSi}_{2} \mathrm{~N}_{4}$ has the same crystal structure with $\mathrm{VSi}_{2} \mathrm{P}_{4}$, and is predicted to be a FMM with the total magnetic moment $0.97 \mu_{B}{ }^{23}$, which is very close to 1.0 $\mu_{B}$. This makes us believe that small strain may induce FMM to FMS transition. Firstly, the ground state of $\mathrm{VSi}_{2} \mathrm{~N}_{4}$ is determined to be $\mathrm{FM}$ in the strain range of $0 \%$ to $11 \%$ by comparing the energy of FM, AFM and NM, and their energy bands are calculated. The total gap of FM $\mathrm{VSi}_{2} \mathrm{~N}_{4}$ as a function of strain using GGA is plotted in Figure 7. It is found that only about $1 \%$ strain can make $\mathrm{VSi}_{2} \mathrm{~N}_{4}$ become FMS, which can be maintained in the strain range of $1 \%$ to $9.5 \%$. When the strain increases, the electronic properties of $\mathrm{VSi}_{2} \mathrm{~N}_{4}$ vary from FMM to SGS to FMS to SGS to FMHM, which is the same with $\mathrm{VSi}_{2} \mathrm{P}_{4}$. The piezoelectric properties of $\mathrm{VSi}_{2} \mathrm{~N}_{4}$ at $3 \%$ strain are studied as a representative. The calculated $C_{11}$ and $C_{12}$ are $431.17 \mathrm{~N} / \mathrm{m}$ and $117.61 \mathrm{~N} / \mathrm{m}$, respectively, and the $e_{11}$ is $6.71 \times 10^{-10} \mathrm{C} / \mathrm{m}$. According to Equation 5, the $d_{11}$ can be attained for $2.14 \mathrm{pm} / \mathrm{V}$.

\section{DISCUSSION AND CONCLUSION}

It is well known that GGA underestimates semiconductor gap, and the hybrid functional HSE06 may give a more appropriate gap. However, for $\mathrm{MoSi}_{2} \mathrm{~N}_{4}$ monolayer, the HSE06 overestimates it's gap. The calculations show that $\mathrm{MoSi}_{2} \mathrm{~N}_{4}$ monolayer is an indirect gap semiconductor with the gap of $1.744 \mathrm{eV}$ (GGA) or $2.297 \mathrm{eV}$ (HSE06), and the experimental value is $1.94 \mathrm{eV}^{22}$. It is found that the GGA gap is more closer to the experimental value than HSE06 one. So, it may be more suitable for $\mathrm{VSi}_{2} \mathrm{P}_{4}$ to use GGA to study it's electronic properties. For $\mathrm{VSi}_{2} \mathrm{P}_{4}$ monolayer, the GGA may underestimates it's gap, but our conclusion should be qualitatively correct, and only the critical points of phase transition change.

We have demonstrated that strain can effectively tune the electronic properties of $\mathrm{VSi}_{2} \mathrm{P}_{4}$ monolayer through first-principles simulations. The results show that FM state in considered strain range is more energetically preferred than AFM and $\mathrm{NM}$ states for $\mathrm{VSi}_{2} \mathrm{P}_{4}$ monolayer. With the increasing strain, the electronic properties of $\mathrm{VSi}_{2} \mathrm{P}_{4}$ vary from FMM to SGS to FMS to SGS to FMHM. The corresponding values of $d_{11}$ of $\mathrm{VSi}_{2} \mathrm{P}_{4}$ in FMS phase for 1\%, $2 \%$ and $3 \%$ strains are 4.61 $\mathrm{pm} / \mathrm{V}, 4.94 \mathrm{pm} / \mathrm{V}$ and $5.27 \mathrm{pm} / \mathrm{V}$, respectively, which are greater than $5 \mathrm{pm} / \mathrm{V}$, a typical value for bulk piezoelectric materials. Similar strain dependence of electronic properties can also be achieved in monolayer $\mathrm{VSi}_{2} \mathrm{~N}_{4}$, and the piezoelectricity and ferromagnetism can also coexist by strain tuning. The $\mathrm{VSi}_{2} \mathrm{P}_{4}$ and $\mathrm{VSi}_{2} \mathrm{~N}_{4}$ may be promising $2 \mathrm{D}$ materials for applications in nanoscale spin electronic devices due to the combination of piezoelectric and magnetic properties.

\section{ACKNOWLEDGMENTS}

This work is supported by the Natural Science Foundation of Shaanxi Provincial Department of Education (19JK0809). We are grateful to the Advanced Analysis and Computation Center of China University of Mining and Technology (CUMT) for the award of CPU hours and WIEN2k/VASP software to accomplish this work.
1 X. Lin, W. Yang, K. L. Wang, W. Zhao, Nat. Electron. 2, 274 (2019).

2 I. Choudhuri, P. Bhauriyal and B. Pathak, Chem. Mater. 31, 8260 (2019).

3 Y. Sun, Z. Zhuo, X. Wu and J. Yang, Nano Lett. 17, 2771 (2017).

4 Y. Ma, Y. Dai, M. Guo, C. Niu, Y. Zhu and B. Huang, ACS Nano, 6, 1695 (2012).

${ }^{5}$ C. Gong, L. Li, Z. Li, H. Ji, A. Stern, Y. Xia, T. Cao, W. Bao, C. Wang, Y. Wang, Z. Q. Qiu, R. J. Cava, S. G. Louie, J. Xia and X. Zhang, Nature 546, 265 (2017).

6 Y. Guo, H. Deng, X. Sun, X. Li, J. Zhao, J. Wu, W. Chu, S. Zhang, H. Pan, X. Zheng, X. Wu, C. Jin, C. Wu and Y. Xie, Adv. Mater. 29, 1700715 (2017).

7 X. M. Wu, Y. L. Feng, S. Li, B. Q. Zhang and G. Y. Gao, J. Phys. Chem. C 124, 16127 (2020).

8 B. Huang, G. Clark, E. Navarro-Moratalla, D. R. Klein, R. Cheng, K. L. Seyler, D. Zhong, E. Schmidgall, M. A. McGuire, D. H. Cobden, W. Yao, D. Xiao, P. Jarillo-
Herrero and X. Xu, Nature 546, 270 (2017).

9 L. Dong, H. Kumar, B. Anasori, Y. Gogotsi and V. B. Shenoy, J. Phys. Chem. Lett. 8, 422 (2017).

10 L. Dong, J. Lou and V. B. Shenoy, ACS Nano, 11, 8242 (2017).

11 Y. Xu, Z. Q. Li, C. Y. He, J. Li, T. Ouyang, C. X. Zhang, C. Tang and J. X. Zhong Appl. Phys. Lett. 116, 023103 (2020).

12 S. D. Guo, X. S. Guo, Z. Y. Liu and Y. N. Quan, J. Appl. Phys. 127, 064302 (2020).

13 M. N. Blonsky, H. L. Zhuang, A. K. Singh and R. G. Hennig, ACS Nano, 9, 9885 (2015).

14 R. X. Fei, We. B. Li, J. Li and L. Yang, Appl. Phys. Lett. 107, 173104 (2015).

15 K. N. Duerloo, M. T. Ong and E. J. Reed, J. Phys. Chem. Lett. 3, 2871 (2012).

16 Y. Chen, J. Y. Liu, J. B. Yu, Y. G. Guo and Q. Sun, Phys. Chem. Chem. Phys. 21, 1207 (2019).

17 W. Wu, L. Wang, Y. Li, F. Zhang, L. Lin, S. Niu, D. 
Chenet, X. Zhang, Y. Hao, T. F. Heinz, J. Hone and Z. L. Wang, Nature 514, 470 (2014).

${ }^{18}$ H. Zhu, Y. Wang, J. Xiao, M. Liu, S. Xiong, Z. J. Wong, Z. Ye, Y. Ye, X. Yin and X. Zhang, Nat. Nanotechnol. 10, 151 (2015).

19 A. Y. Lu, H. Zhu, J. Xiao, C. P. Chuu, Y. Han, M. H. Chiu, C. C. Cheng, C. W. Yang, K. H. Wei, Y. Yang, Y. Wang, D. Sokaras, D. Nordlund, P. Yang, D. A. Muller, M. Y. Chou, X. Zhang and L. J. Li, Nat. Nanotechnol. 12, 744 (2017).

${ }^{20}$ M. Dai, Z. Wang, F. Wang, Y. Qiu, J. Zhang, C. Y. Xu, T. Zhai, W. Cao, Y. Fu, D. Jia, Y. Zhou, and P. A. Hu, Nano Lett. 19, 5416 (2019).

21 J. H. Yang, A. P. Wang, S. Z. Zhang, J. Liu, Z. C. Zhong and L. Chen, Phys. Chem. Chem. Phys., 21, 132 (2019).

${ }^{22}$ Y. L. Hong, Z. B. Liu, L. Wang et al., Science 369, 670 (2020).

23 L. Wang, Y. P. Shi, M. F. Liu et al., arXiv:2008.02981 (2020).

24 S. D. Guo, Y. T. Zhu and W. Q. Mu, arXiv:2008.05751 (2020).

25 P. Hohenberg and W. Kohn, Phys. Rev. 136, B864 (1964);
W. Kohn and L. J. Sham, Phys. Rev. 140, A1133 (1965).

26 J. P. Perdew, K. Burke and M. Ernzerhof, Phys. Rev. Lett. 77, 3865 (1996).

27 G. Kresse, J. Non-Cryst. Solids 193, 222 (1995).

28 G. Kresse and J. Furthmüller, Comput. Mater. Sci. 6, 15 (1996).

29 G. Kresse and D. Joubert, Phys. Rev. B 59, 1758 (1999).

30 X. Wu, D. Vanderbilt and D. R. Hamann, Phys. Rev. B 72, 035105 (2005).

31 E. Scalise, M. Houssa, G. Pourtois, V. Afanas'ev and A. Stesmans, Nano Res. 5, 43 (2012).

32 H. K. Liu, G. Z. Qin, Y. Lin and M. Hu, Nano Lett. 16, 3831 (2016).

33 N. Jena, Dimple, S. D. Behere and A. D. Sarkar, J. Phys. Chem. C 121, 9181 (2017).

34 Dimple, N. Jena, A. Rawat, R. Ahammed, M. K. Mohanta and A. D. Sarkar, J. Mater. Chem. A 6, 24885 (2018).

35 S. D. Guo and B. G. Liu, J. Phys.: Condens. Matter 24, 045502 (2012).

36 R. C. Andrew, R. E. Mapasha, A. M. Ukpong and N. Chetty, Phys. Rev. B 85, 125428 (2012). 